\title{
Overexpression of Neutrophil MMP-9 and HIF-I $\alpha$ May Contribute to the Finger-Like Projections Formation and Histo-Pathogenesis in Nasal Inverted Papilloma
}

Tao Li, (D) ${ }^{1,2}$ Kai Sen Tan, (iD ${ }^{2-5}$ Yan Yi Tu, (D) ${ }^{1,2}$ Li Zhao, (D) ${ }^{6}$ Jing Liu, (D) ${ }^{2,3}$

Hsiao Hui Ong, ID $^{2,3}$

De Yun Wang, (iD) ${ }^{2,3}$ Li Shi (D)

'Department of Otolaryngology-Head and Neck Surgery, Shandong Provincial ENT

Hospital, Cheeloo College of Medicine, Shandong University, Jinan, People's Republic of China; ${ }^{2}$ Department of Otolaryngology, Yong Loo Lin School of Medicine, National University of Singapore, Singapore, Singapore; ${ }^{3}$ National University Health System Infectious Diseases Translational Research Program, Yong Loo Lin School of Medicine, National University of Singapore, Singapore, Singapore; ${ }^{4}$ Department of Microbiology and Immunology, Yong Loo Lin School of Medicine, National University of Singapore, Singapore, Singapore; ${ }^{5}$ Biosafety level 3 Core Facility, Yong Loo Lin School of Medicine, National University Health System, National University of Singapore, Singapore, Singapore; ${ }^{6}$ Department of Otolaryngology, The Second Hospital of Shandong University, Shandong University, Jinan, People's Republic of China

Correspondence: Li Shi

Department of Otolaryngology, Shandong Provincial ENT Hospital, Cheeloo College of Medicine, Shandong University,

Duanxing West Road, Jinan, Shandong,

250033, People's Republic of China

Tel +8653183086279

Fax +86 531 87980304

Email shilil26@sina.com

De Yun Wang

Department of Otolaryngology, Yong Loo Lin School of Medicine, National

University of Singapore, IE Kent Ridge

Road, Singapore, I1 9228

Tel +6567725373

Fax +6567753820

Email entwdy@nus.edu.sg
Background: Nasal inverted papilloma (NIP) is defined based on its histological characteristic of inverted epithelium growth into the stroma. The inversion can result in epithelial growth in the underlying connective tissue stroma when the basement membrane completely separates from the epithelial layer. To date, such inversion mechanism underlying NIP's pathological phenomenon is unknown. Therefore, we hypothesized that mediators and soluble proteins released by neutrophils, the most predominant infiltrating cells in NIP, is vital in causing the epithelial changes and pathogenesis of NIP.

Methods: We collected 37 NIP tissues from patients who underwent surgical removal of NIP and performed hematoxylin-eosin (HE), immunohistochemical, and immunofluorescence staining to analyze in-depth the basic characteristics of NIP, including detecting the expression and distribution of MMPs and associated factors in NIP. Western blotting and quantitative real-time PCR were further performed to analyze the protein and mRNA expression levels of specific factors including MMPs, HIF-1 $\alpha$, and tissue inhibitors of metalloproteinases (TIMPs).

Results: We observed finger-like projections that insert into the epithelium in NIP tissue as its main characteristics. The projections contain fibroblasts, extracellular matrix, capillaries, and infiltrating inflammatory cells. We found abundant neutrophils clustered at the finger-like projection of NIP, and also noted MMP-1 and MMP-9 were up-regulated in NIP ( $p<0.05)$, whereas TIMP-1/3 was decreased. The expression level of HIF-1 $\alpha$ was also found to be increased in NIP tissue. We further showed that MMP-9 and HIF-1 $\alpha$ were mainly expressed by neutrophils and were predominantly observed in the finger-like projections that contribute to the NIP pathology.

Conclusion: Upregulation and release of MMP-9 and HIF-1 $\alpha$ from infiltrating neutrophils may cause damage to the epithelial basement membrane and epithelial clefts, forming fingerlike projections with angiogenesis and fibroblasts insertion, resulting in epithelial growth in the tissue stroma, a typical histo-pathological characteristic in NIP.

Keywords: nasal inverted papilloma, finger-like projection, MMP-9, HIF-1 $\alpha$, pathogenesis, neutrophils

\section{Introduction}

Nasal inverted papilloma (NIP) is a benign tumor that occurs in the nasal cavity and paranasal sinuses. Representing $0.5-4 \%$ of nasal tumors, NIP has a high recurrence rate, and $5-15 \%$ of NIP progress into squamous carcinoma. ${ }^{1,2}$ Conventionally, NIP is 
a kind of neoplasm arising from the Schneiderian membrane, ${ }^{3}$ and its histopathological characteristics include i) epithelium inverting into the stroma, where the basement membrane completely separates the epithelial component into the underlying connective tissue stroma; ${ }^{4}$ ii) increase thickness of the epithelium with squamous metaplasia; iii) increase inflammatory cells' infiltration (mostly neutrophil, macrophage, lymphocyte), mainly distributed at the epithelium and tissue stroma; ${ }^{5}$ iv) small original NIP initiation site compared to the tumor body. In NIP tissue, the stroma projected into epithelium were frequently described as "finger-like projections" in articles describing inverted papilloma generated from other parts of the body, including the breast intraductal, esophagus, gastric, and cervix. ${ }^{6-8}$ With the available literature, the histopathological characteristics of NIP are well documented; the mechanisms driving the formation of finger-like projections altering and inserting the epithelium into the tissue stroma as part of NIP pathogenesis, however, are understudied.

MMPs are a family of zinc-dependent endopeptidases that catalyzes proteolytic activities to aid the breakdown of extracellular matrixes (ECM). ${ }^{9}$ MMPs are expressed mainly in neutrophils, lymphocytes, macrophages, fibroblasts, and epithelial cells; and specifically in macrophage and epithelial cells for MMP-7; as well as fibroblasts and macrophages for MMP- $1 .{ }^{10}$ Activated MMPs are implicated in many physiological functions such as tissue remodeling, wound healing, inflammatory process, and communication between the epithelium and tissue stroma. ${ }^{11-13}$ In recent years, studies have also found that MMPs could influence the tissue microenvironment by disrupting the equilibrium between proliferative and anti-proliferative signals. ${ }^{14}$ Under physiological conditions, the expression and activity of MMPs are precisely regulated; however, they are commonly found to be up-regulated in pathological conditions. ${ }^{13}$

Among the MMPs, MMP-9 is a member of gelatinases that can digest the extracellular matrix, laminin, elastin, and vitronectin, ${ }^{15,16}$ and is established to be more effective in degrading basement membranes than other MMPs. ${ }^{17}$ In many diseases, secreted MMP-9 damages the basement membrane, allowing neutrophils, dendritic cells, and eosinophils to infiltrate into the epithelium. ${ }^{18-20}$ Our previous study has found that in NIP, neutrophils made up about $54.3 \%$ of the infiltrating cells; while macrophages amount to about $12.2 \% .^{5}$ As MMP-9 is a main secretory product by neutrophils, it may play a role in NIP pathogenesis. So far, studies associating MMP-9 to NIP pathogenesis are rare, and MMP-9 expression did vary when compared to healthy controls. ${ }^{21,22}$ Besides, MMP-9 could also be induced by stress factors such as HIF-1 $\alpha$ to promote the process of angiogenesis and epithelial remodeling. ${ }^{23}$ The expression level of HIF-1 $\alpha$ in NIP remains unclear. Therefore, this study aimed to investigate the expression levels and pathogenic role of MMP-9 (as compared to other MMPs) and HIF-1 $\alpha$ 's to elucidate their role in initiating and progressing NIP pathogenesis.

\section{Materials and Methods Study Samples}

All NIP ( $\mathrm{n}=37$ ) samples were collected from patients who were diagnosed with NIP by a pathologist and have undergone endoscopic tumor resection at the Department of Otolaryngology in Qilu Hospital of Shandong University and The Second Hospital of Shandong University. Inferior Turbinate $(n=24)$ of healthy controls were obtained from patients undergoing corrective surgery for septal deviation or other indications like epistaxis, nasal bone fracture, which does not have acute or chronic inflammatory diseases present. All patients provided informed consent and patients' characteristic are shown in Table 1.

\section{H\&E and Immunohistochemical Study}

$4 \%$ Paraformaldehyde solution was used to fix fresh tissue samples, which were then embedded in paraffin. Paraffin sections were cut at a thickness of $2 \mu \mathrm{m}$ for Hematoxylin and eosin (H\&E) staining and immunohistochemical (IHC) staining. Evaluation of NIP histological characteristics and determination of basement membrane thickness was performed via $\mathrm{H} \& \mathrm{E}$ staining. The distribution of MMPs and neutrophils was detected by IHC. Briefly, sections were incubated with $1 \mathrm{X} \mathrm{pH} 6.0$ antigen retrieval buffer (Abcam, USA) and 3\% hydrogen peroxide (Sigma Aldrich, USA) for eliminating endogenous peroxidase.

Table I Clinical Characteristics of NIP Patients

\begin{tabular}{|l|c|c|}
\hline & Healthy Control & NIP \\
\hline Sample size & 24 & 37 \\
Age, years & $27.6(17-5 I)$ & $46.6(28-72)$ \\
Gender, male/female & $15 / 9$ & $32 / 5$ \\
Smoking, smoker/nonsmoker & $3 / 21$ & $9 / 28$ \\
First diagnose of NIP & 0 & 23 \\
Recurrent NIP & 0 & 14 \\
Neutrophilia & 5 & $37^{* * *}$ \\
\hline
\end{tabular}

Notes: A sample was considered as having neutrophilia when the percentage of neutrophils is over 10\%; Unpaired student t-text was performed to analyze the significance $(p), * * * p<0.001$. 
Sections were then blocked in 10\% goat serum (Abcam, USA) and incubated overnight in the dark at $4^{\circ} \mathrm{C}$ with primary antibodies of MMP-1 (1:200, Abcam, ab137332), MMP-7 (1:200, Abcam, ab207229), MMP-9 (1:200, Abcam, ab76003), TIMP-1 (1:200, Abcam, ab211962), TIMP-3 (1:200, Abcam, ab39184), neutrophil elastase (1:1000 clone NP57 Dako, Glostrup, Denmark), HIF-1 $\alpha$ (1:200, Abcam, ab179483). Next, all sections were incubated with the Dako EnVision1 System-HRP (Dako) at room temperature for 30 minutes. For color development, Diaminobenzidine was used as a substrate, and all sections were counterstained by hematoxylin.

\section{Immunofluorescences}

To investigate MMPs' distribution in NIP, we performed immunofluorescence staining on NIP tissue sections. After antigen retrieval and antigen blocking. We co-stained MMP-9 and P63 (1:400, Abcam, ab32353) to check the MMP-9 localization and its relationship with the basement membrane of the NIP epithelium. Double staining of alphasmooth muscle actin (1:200, Abcam, ab7817) and P63 was performed to detect if the finger-like projections contain fibroblasts and capillaries. Co-staining of MMP-9 with neutrophil elastase (1:1000 clone NP57 Dako, Glostrup, Denmark), anti-mast cell antibody (1:200, Abcam, ab134932), ECP (1:200, Abcam, ab1907715), CD4 (1:200, Abcam, ab33775), FOXP3 (1:200, Abcam, ab20034), were performed to determine the cell type that secretes MMP-9. All primary antibodies were incubated at $4^{\circ} \mathrm{C}$ in the dark overnight, followed by 3 times PBS wash at 5 minutes each. Sections were then incubated with fluorescence secondary antibody (1:500, Abcam, ab150077, ab150116), mounted with DAPI (P36931, Invitrogen), and viewed with a fluorescence microscope (Olympus, Japan).

\section{Western Blotting}

Protein was extracted from fresh NIP tissue using ice-cold RIPA lysis buffer (Abcam) mixture containing protease and phosphatase inhibitor (Sigma Aldrich). After 30 minutes of incubation on ice with shaking, followed by centrifugation at $14,000 \mathrm{rpm}$, the supernatant containing the protein was aspirated for use in Western blot analysis. Protein concentration was first determined using BCA protein assay (Thermo Fisher, USA); then, 4X laemmli sample buffer was added to the protein samples according to the manufacturer's instructions, and heated at $95^{\circ} \mathrm{C}$ for 10 minutes. $30 \mu \mathrm{g}$ total protein from each sample was subjected to electrophoresis in $10 \%$ SDS-PAGE gel and then transferred onto a $0.22 \mu \mathrm{m}$
PVDF membrane (Millipore, USA). After 2 hours nonspecific blocking with 5\% skimmed milk, primary antibody of MMP-1 (1:2000, Abcam, ab137332), MMP-7 (1:2000, Abcam, ab207229), MMP-9 (1:4000, Abcam, ab76003), TIMP-1 (1:2000, Abcam. Ab109125), TIMP-3 (1:2000, Abcam, ab39184), GAPDH (1:5000, Abcam, ab186930) were added to the membrane and incubated at $4^{\circ} \mathrm{C}$ overnight. HRP-conjugated anti-rabbit IgG or anti-mouse IgG were then added as secondary antibody at room temperature for 1 hour. After secondary antibody staining, ECL reagent (BioRad, USA) was used to visualize the blots. Densitometry was performed with ImageJ software to quantify the protein amounts of each sample.

\section{RNA Extraction and Real-Time PCR}

To determine the mRNA level of MMPs and TIMPs, realtime quantitative PCR was performed. All primers used in the study were pre-designed primers from Sigma Aldrich, and the primer sequences are presented in Supplementary Table 1. Total RNA was extracted from NIP tissue $(n=37)$ and healthy control $(n=24)$ using mirVana miRNA isolation kit (Invitrogen, USA), and then reversed-transcribed into cDNA by qScript cDNA Supermix (Quanta Bio, USA). The mRNA levels of MMP-1, MMP-2, MMP-7, MMP-9, TIMP-1, TIMP-3 were then detected using GoTaq Green Master Mix (Promega). Real-time quantitative PCR was performed using ABI ViiA 7 Real-Time PCR System (Applied Biosystems). Relative expression was calculated using the comparative $2^{-\Delta \Delta \mathrm{Ct}}$ methods.

\section{Statistical Analysis}

All data were analyzed using GraphPad Prism 8 and shown as mean $\pm \mathrm{SD}$, with $\mathrm{n}$ numbers stated individually for each experiment. The Student's $t$-test was used as the statistical test in comparing results between the NIP and control groups.

\section{Results}

\section{Neutrophils, Fibroblasts, and Capillaries Were Clustered at the Finger-Like Projections in NIP Tissue}

We first use hematoxylin-eosin staining to observe the histopathological characteristics of NIP tissue. Through the HE staining (Figure 1A and Supplementary Figure 1) of the NIP sections $(n=37)$, we observed many finger-like projections were inserted into the epithelium, where the composition of the finger-like projections includes inflammatory cells and capillaries (red triangle). Neutrophil 

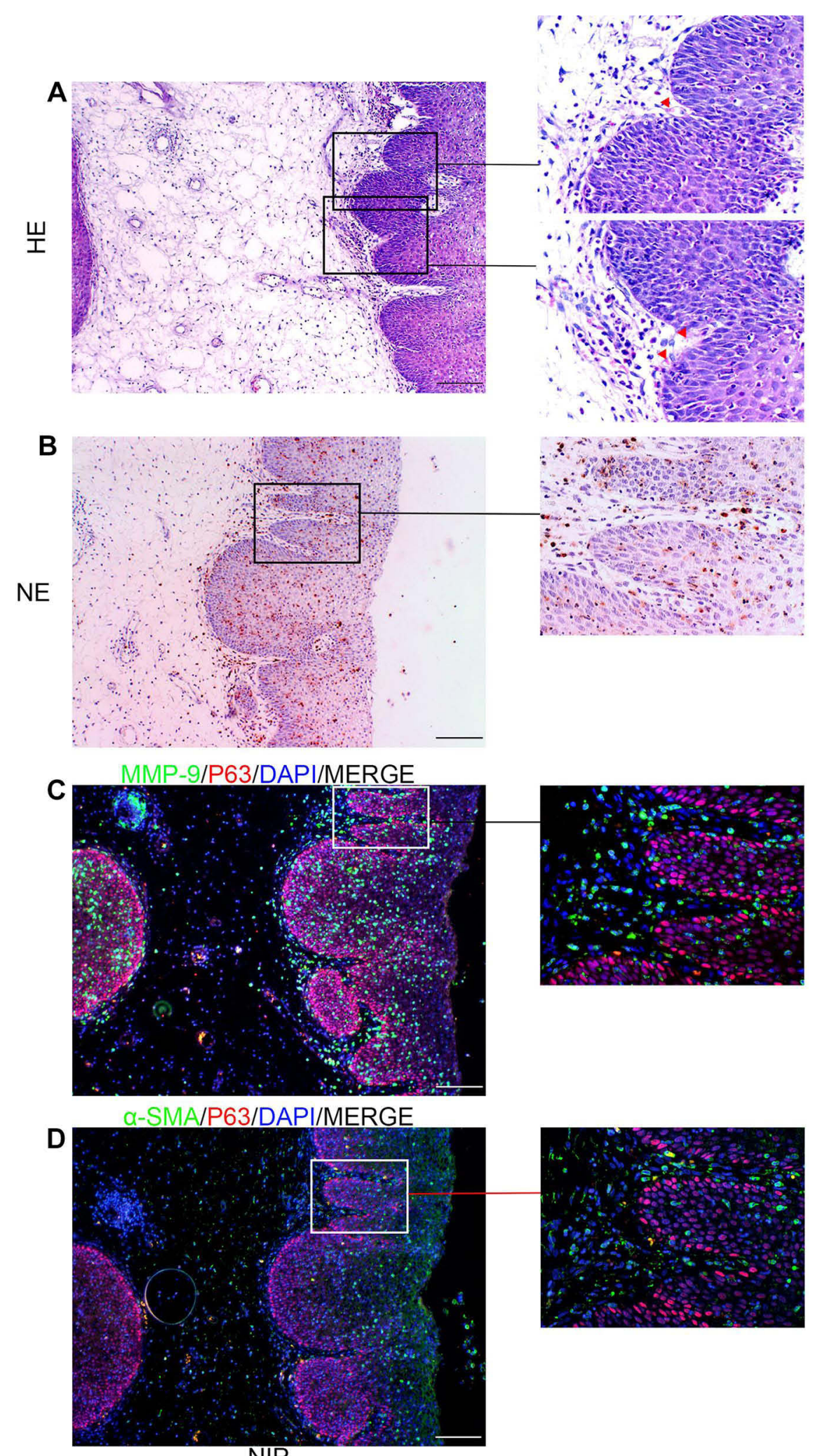

NIP

Figure I Basic characteristics of NIP. (A) H\&E staining of nasal inverted papilloma in I00x and 400x magnifications showing finger-like projections inserted into epithelium (red triangles). $(\times 100$ magnification scale bar $=100 \mu \mathrm{m}, \times 400$ magnification scale bar $=20 \mu \mathrm{m})$. (B) Immuno-histochemical assay of neutrophil elastase, indicating neutrophils in finger-like projections. ( $\times 100$ magnification scale bar $=100 \mu \mathrm{m}, \times 400$ magnification scale bar $=20 \mu \mathrm{m})$. $(\mathbf{C}$ and $\mathbf{D})$ Double staining of MMP-9 with P63 and $\alpha-S M A$ with P63, indicating the distribution of MMP-9 near the basement membrane and showed fibroblasts insertion at the finger-like projections $(\times 400$ magnification scale bar $=20 \mu \mathrm{m})$. Red triangles indicate capillaries. Abbreviations: NE, neutrophil elastase; $\alpha$-SMA, $\alpha$-smooth muscle actin. 
elastase is a specific marker of neutrophils and $\alpha$-SMA is a marker of fibroblasts. As shown in Figure 1B, we observed that majority of the inflammatory cells present were neutrophils, distributed in the stroma near the epithelium in projections. Figure 1C shows that MMP-9 was found at the tissue stroma near the epithelium. Figure 1D indicates that $\alpha$-SMA positive cell was located in fingerlike projections, which means fibroblasts are inserted in Finger-like projections in nasal inverted papilloma. As presented in Supplementary Figure 1, a black arrow in case- 2 and case-3, indicated the presence of isolated, island-like epithelium region was also found segmented in the stroma. Supplementary Figure 2A and B further showed neutrophils (stained positive with neutrophil elastase) clustering at the boundaries between the epithelium and stroma near the basement membrane, as well as at the epithelial islands in the sub-epithelium, overlapping with MMP-9 staining. The clusters were especially prominent in the finger-like projections, where obvious aggregation of neutrophils was observed. Also, as shown in Supplementary Figure $3 \mathrm{~A}$ and $\mathrm{B}$, the number of neutrophils in the finger-like projections was also observed to be significantly higher than that of the flat area.

\section{NIP Basement Membrane is Thinner, Less Intact Than Healthy Controls}

In NIP tissue, the basement membrane separates the epithelium from the stroma. However, the thickness of the basement membrane appeared abnormal when we compared tissue sections of nasal inverted papilloma with healthy control (Figure 2A). We found that the basement membrane of NIP tissue was significantly thinner when compared to healthy control (Figure 2B). In addition, Figure 2A and Supplementary Figure 4 also showed that the NIP basement membrane was less intact, with red arrows indicating broken regions of the basement membrane where the MMP-9 clusters in the finger-like projections were observed. The thinning and breaking of the basement membrane may be due to the infiltration and secretion from these infiltrating cells such as MMPs, which serve to digest the basement membrane initiating remodeling processes.

\section{MMPs Were Up-Regulated in Nasal Inverted Papilloma}

To investigate the expression levels of MMPs and their inhibitor TIMPs in nasal inverted papilloma, we performed Western blotting and Real-time quantitative PCR to compare their expression in healthy control and NIP tissue. Figure 3A reveals that MMP-1 and MMP-7, but not MMP-9, were mainly expressed in the epithelial cells of the NIP, Supplementary Figures $2 \mathrm{~B}$ and $\underline{3}$ further indicated that MMP-9 was instead localized to the infiltrating cells at infiltration sites near the basement membrane, and is especially clustered in the regions with finger-like projections than flat regions of the NIP stroma (Supplementary Figure 4B). Western blotting and quantification (Figure 3B-E) further showed significantly increased protein levels of MMP-1, MMP-7, and MMP-9. In contrast, the MMP inhibitors TIMP-1 and TIMP-3 decreased significantly in NIP tissue (Figure 3B, F and G). In addition, as shown in Figure 4A-F, the mRNA levels generally corroborate with the protein expression in which we observed that MMP-1 and MMP-7, but not MMP-9, were significantly increased in NIP; while MMP-2, TIMP-1, and TIMP-3 mRNA were decreased in NIP. The lack of MMP-9 mRNA increase may be due to their localization to infiltrating cells, but not epithelium, in which transcription activity was reduced.

\section{MMP-9 in NIP Tissue Was Mainly Secreted by Neutrophils}

So far, we showed that increased MMP-1 and MMP-7 were a result of expression by the epithelial cells. On the other hand, the source of MMP-9 in NIP remains to be confirmed. Therefore, to ascertain the infiltrating cell type that serves as the main source of MMP-9 in NIP, costaining of MMP-9 with neutrophils elastase, CD68, and ECP, MAST, CD4, FOXP3 were performed in NIP tissue (Figure 5A-F). Neutrophil elastase is a specific marker of neutrophils, CD68 is the marker of macrophages; while ECP is the marker of eosinophils, MAST is the marker of mast cells, CD4 and FOXP3 are the marker of $\mathrm{CD} 4{ }^{+}$ $\mathrm{T}$ cells and $\mathrm{FOXP}^{+} \mathrm{T}$ cells. As shown in Figure 5 and Supplementary Figure 5, we discovered that neutrophils were the main source of MMP-9 in NIP, where the most co-localization of MMP-9 with neutrophil elastase was observed, corroborating with the high neutrophil elastase positive staining in the previous results (Figure 5A). Even though macrophages were also found to produce MMP-9, the numbers of macrophages were much lesser than neutrophils in NIP (Figure 5B). Besides, the distribution of macrophages also did not show a strong co-localization with the finger-like projections. The Co-staining of MMP9 with CD4 (T-cells), FOXP3 (activated T-cells), and MAST (mast cells) showed that their production of 

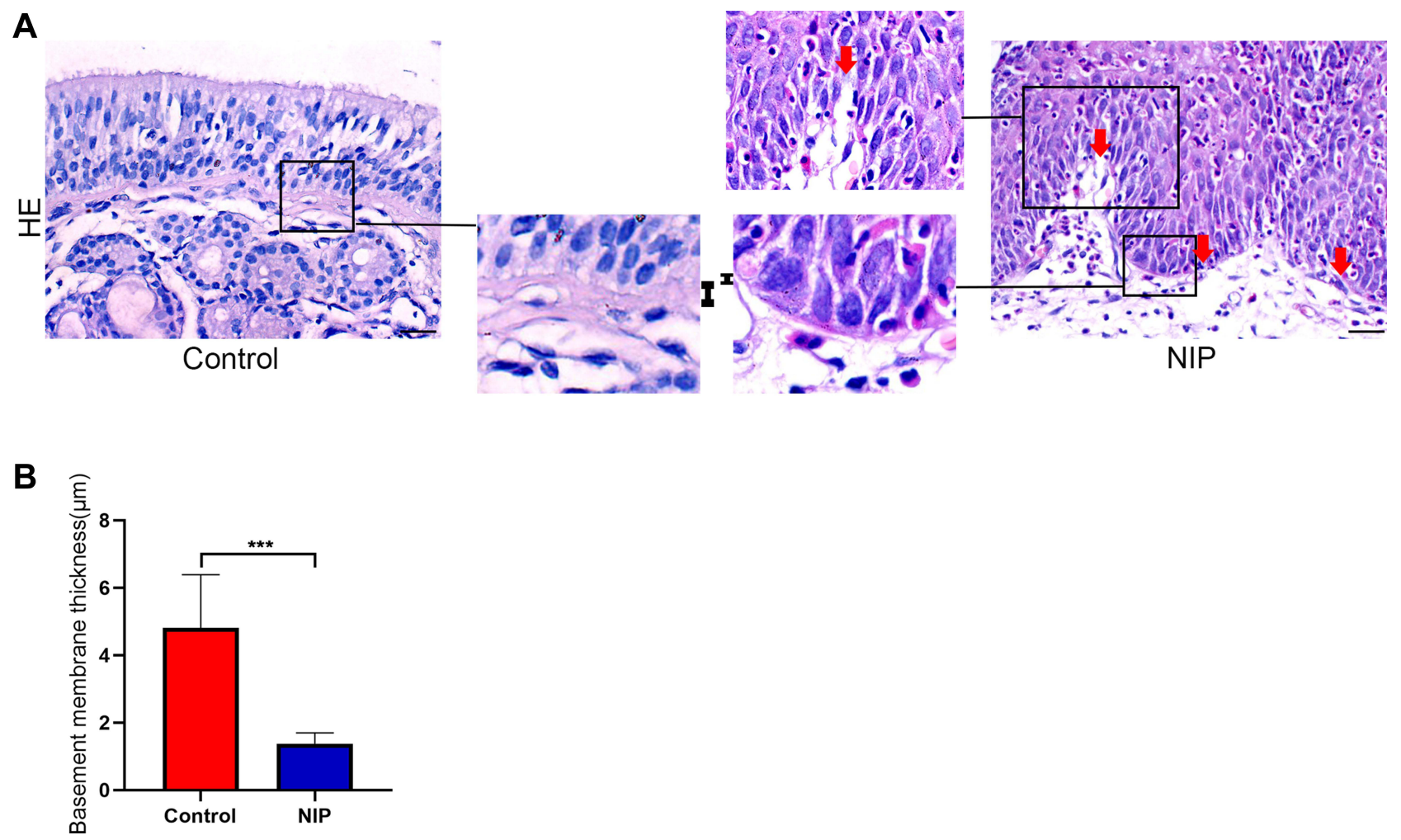

Figure 2 Basement membrane thickness of NIP tissue and healthy control. (A) H\&E staining was performed to determine the thickness of NIP and healthy control tissue. "I" represents the thickness of basement membrane. Red arrow indicates broken area of NIP epithelium. (B) Measurement of epithelial basement membrane thickness by ImageJ (healthy control $n=12$, NIP $n=14$ ). The bar graph showed average quantification of membrane thickness and student's $t$-test was performed to determine the statistical significance between groups. Data were presented as mean \pm SD. $* * * p<0.001$.

MMP-9 was also not as high as compared to in neutrophils. The strong presence of neutrophils at finger-like projections in NIP tissue suggested that their main product, MMP-9, is crucial in the formation of the pathological changes in NIP, compared to other MMPs of other origins.

\section{The Expression Level of HIF-I $\alpha$ Was Increased in NIP}

$\mathrm{HIF}-1 \alpha$ is a hypoxic factor that can promote angiogenesis. As we observed an increased number of capillaries in NIP tissue, we thus further determined the expression level of HIF- $1 \alpha$ in NIP tissue. Figure 6A and B indicated that the production of HIF-1 $\alpha$ in NIP tissue was up-regulated. Interestingly, HIF-1 $\alpha$ and MMP-9 are both expressed by neutrophils, as shown with their co-localization with neutrophil elastase (Figure 6C and D, Supplementary Figure 6). HIF-1 $\alpha$ was also found to be expressed in the finger-like projections and may likely induce the capillaries' formation in the finger-like projections.

\section{Discussion}

Due to its unique histopathological characteristics, NIP is relatively easier to diagnose in clinical management, despite the many gaps in understanding in its pathogenesis. In this study, we systematically analyzed the expression and distribution of factors associated with NIP, with MMP-9 and HIF$1 \alpha$ being the primary focus for their contribution towards NIP pathogenesis. As shown in Figure $7 \mathrm{~A}-\mathrm{C}$, we speculate that with persistence viral or bacterial invasion, and other inflammatory mediators, a large amount of neutrophils may cluster in the schneiderian membrane and secretes MMP-9 and HIF$1 \alpha$ to dissolve basement membrane, promoting capillary and finger-like projection formation. Our study first described the special characteristics of NIP in their pathogenesis mechanism in the upper airway epithelium. We also described that neutrophil infiltration and its secreted factors may be vital in 
A

NIP (Finger-Like Projections Area)
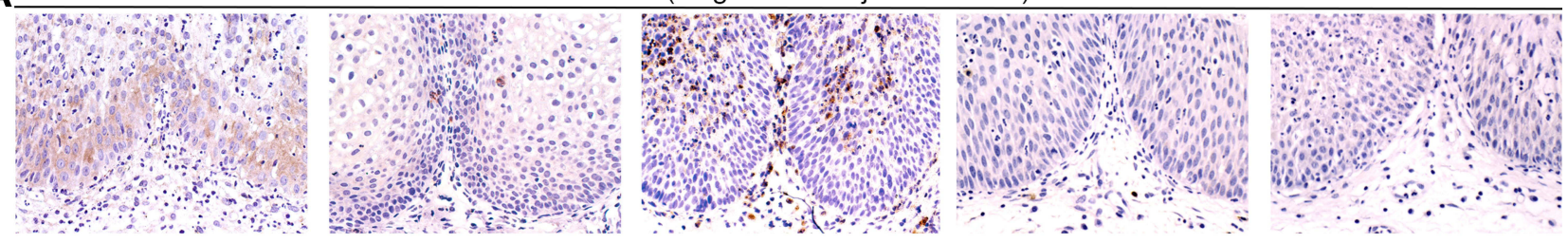

NIP(Flat area)
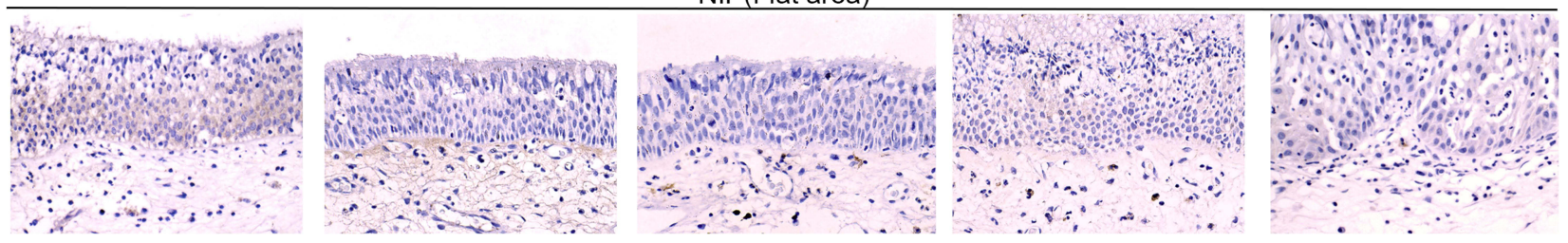

Control

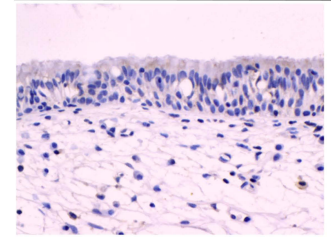

MMP-1

B

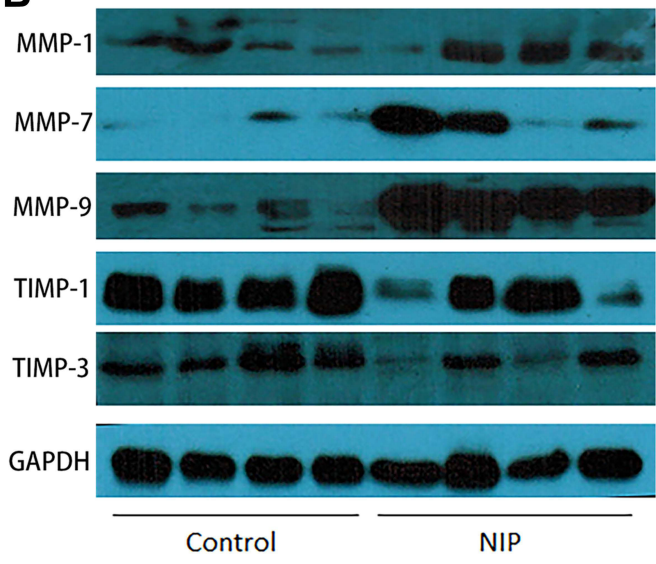

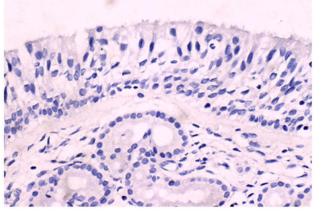

MMP-7

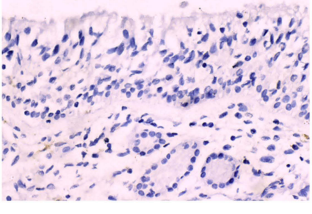

MMP-9
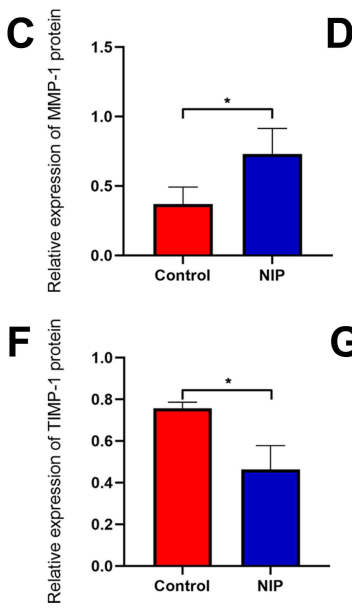

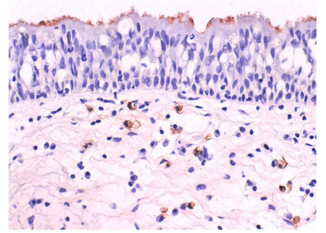

TIMP-1

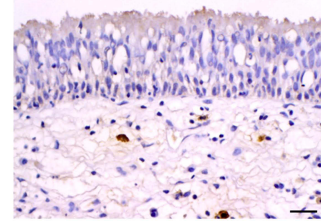

TIMP-3
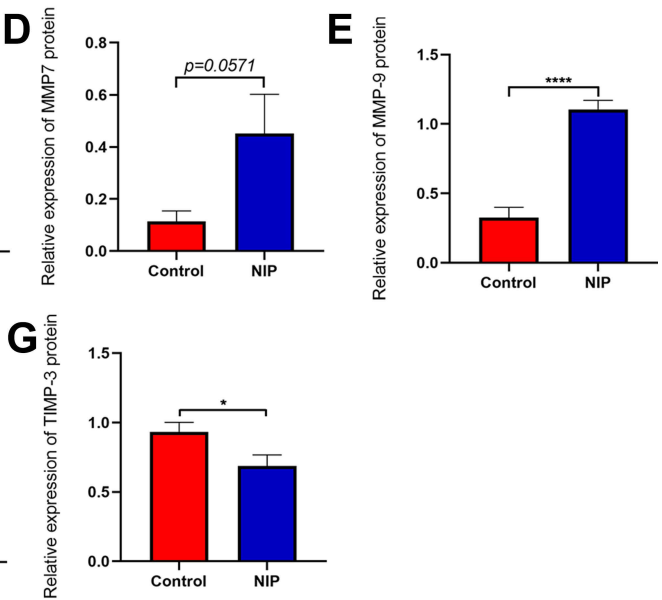

Figure 3 Expression and distribution of MMP-I/7/9 and their inhibitors TIMP-I/3 in NIP tissue. (A) immunohistochemical staining for MMP-I/7/9, TIMP-I/3 in nasal inverted papilloma, all pictures were shown as $\times 400$ magnification (scale bar $=20 \mu \mathrm{m}$ ). (B) Representative Western blotting image for MMP-I/7/9, TIMP-I/3 and housekeeping GAPDH from total protein extracted from NIP and healthy control tissue (healthy control $n=8, \mathrm{NIP} n=8)$. $(\mathbf{C}-\mathbf{G})$ MMP-I/7/9, TIMP-I/3 relative protein expression levels in healthy control and NIP tissue, the data were analyzed using ImageJ and shown as mean \pm SD. Statistical significance was analyzed using student's $t$-test, $* p<0.05$, $* * * * p<0.0001$.

Abbreviations: FLP, finger-like projections; Flat, area of NIP epithelium without finger-like projections.

NIP formation, which presents a potential target in the clinical management of NIP.

From the cell type analysis of NIP tissue in our study, we found that neutrophils are the main infiltrating cells in the stroma and basement membrane of NIP. While neutrophil is a key member of the innate immune cells, dysregulation of neutrophil infiltration may lead to tissue damage in chronic inflammations. ${ }^{24}$ When responding to threats, neutrophils infiltrate the inflammation site by generating factors that dissolve the ECM and basement membrane. In a chronically inflamed site where neutrophils are abnormally elevated, these factors will excessively damage ECM and basement membrane leading to injuries in the stromal matrix. ${ }^{25}$ Conversely, the ECM could also affect neutrophil apoptosis indirectly through the tumor necrosis factor-alpha (TNF- $\alpha$ ) pathway, releasing reactive oxygen species that may further contribute to inflammation and remodeling. ${ }^{26}$ These interactions between neutrophils and the ECM resulted in repeated damage and re-construction in the epithelium potentiating 
A

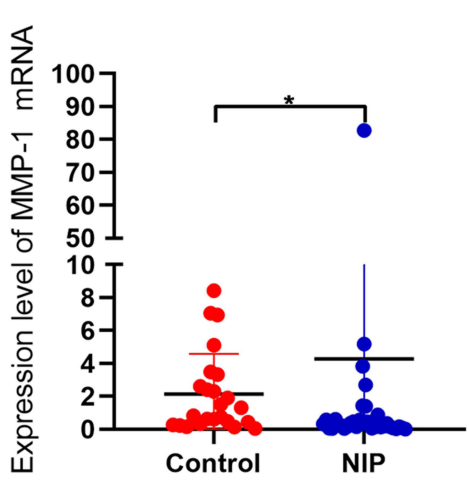

D

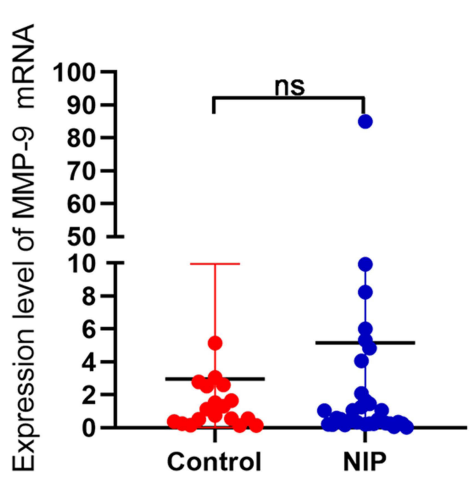

B

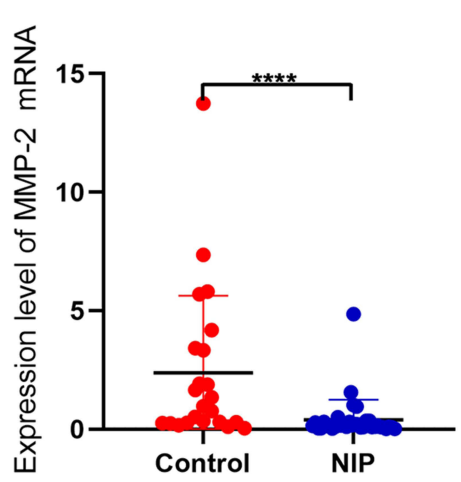

E

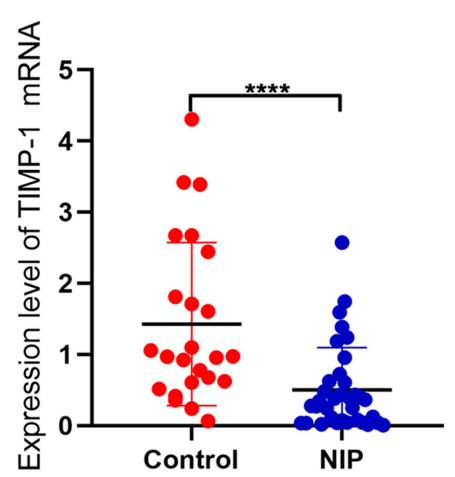

C

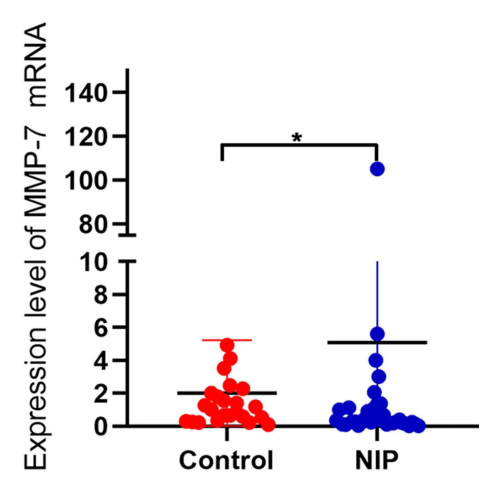

$\mathbf{F}$

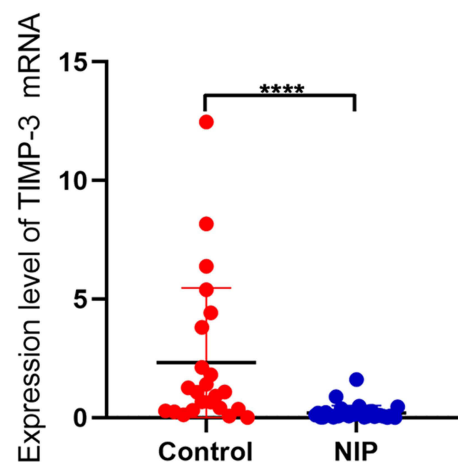

Figure 4 mRNA expression levels of MMPs and associated factors in NIP and healthy control tissue. (A-F) mRNA levels of MMP-I, MMP-2, MMP-7, MMP-9, TIMP-I, TIMP3 were detected by qRT-PCR, and analyzed with GraphPad 8. Data were shown as mean \pm SD and statistical significance was analyzed using student's $t$-test, $* p<0.05$, $* * * * p<0.0001$

Abbreviation: ns, not significant.

NIP formation. In addition, macrophages were also found to be elevated in NIP tissue. The macrophages can be divided into M1 and M2 type macrophages, with M1 macrophages predominantly carrying out proinflammatory roles removing external threat; while M2 macrophages maintain the homeostasis of the inflammatory microenvironment via anti-inflammatory cytokines and scavenging dead immune cells at the inflammation site. Our previous study showed that in NIP, the ratio of macrophage/neutrophils is much lower compared to the healthy controls, ${ }^{5}$ indicating potential dysregulation in inflammatory homeostasis and clean up of inflammatory sites. The reduced rate of resolving inflammation may lead to apoptosis of the increased number of neutrophils at the site, releasing its contents that perpetuate ECM damage and cause secondary infections that may prolong inflammatory processes contributing to NIP formation. ${ }^{27}$

In this study, we further identify secretory factors that may contribute to ECM damage and remodeling processes that may lead to NIP formation and pathogenesis. We found that clustered neutrophils at the stroma, particularly at the finger-like projection region, carries and secretes
MMP-9 and HIF-1 $\alpha$. These factors in turn caused degradation of the basement membrane and promote angiogenesis which resulted in the insertion of fibroblasts and capillaries into the epithelium through the broken basement membrane, further squeezing and distorting the epithelium. In addition, while the remodeling processes occur, the continuous proliferation of the epithelium, fibroblasts, and capillaries resulted in the unclear separation of the epithelium and promotes the development of NIP in various forms. As shown in Supplementary Figure 1, some parts of the epithelium may be cut by fibroblasts and capillaries to form epithelial islands, while most of the epithelium is located on the surface of the tumor. Due to the long-term existence of chronic inflammation and pathogenic factors, the cutting and insertion occur continuously in NIP, leading to the unique pathological characteristics of nasal inverted papilloma (Figure 7A-C).

Another important function of MMP-9 is to promote angiogenesis through the VEGF-dependent pathway. ${ }^{28}$ MMP-9 can be expressed in the blood vessel endothelial cells and loose tight junction, to facilitate the movement of inflammatory cells into the stroma. At the same time, the 

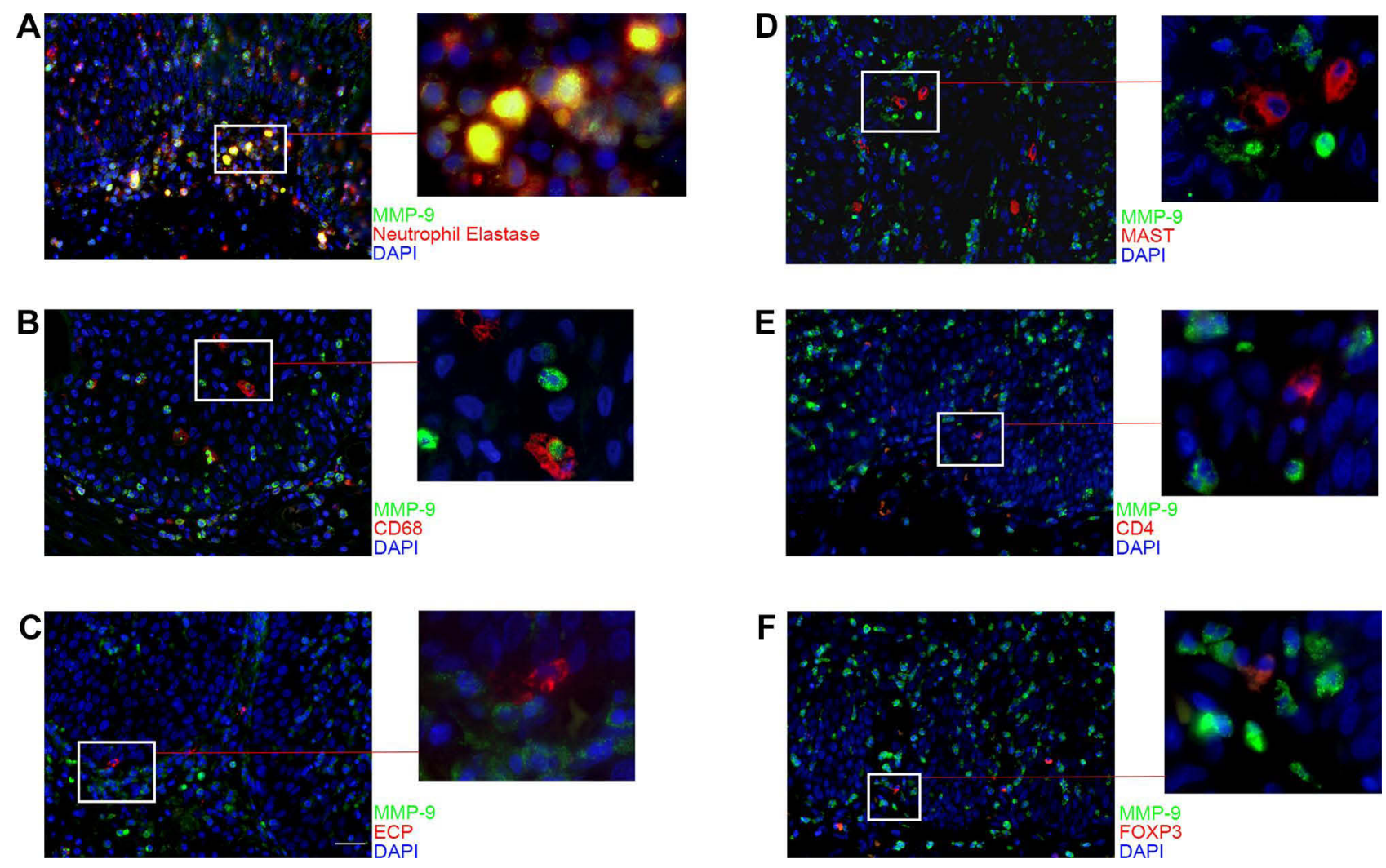

Figure 5 Distribution of MMP-9 in infiltrating cells of NIP tissue. (A-F) immunofluorescence co-staining for MMP-9 (green) with neutrophil elastase, CD68, ECP, MAST, CD4, or FOXP3 (red) were performed to detect which cell type secrete MMP-9. Each co-staining was presented with $\times 400$ magnification $($ scale bar $=20 \mu m$ ). Higher magnification photographs were presented on the right side of the images.
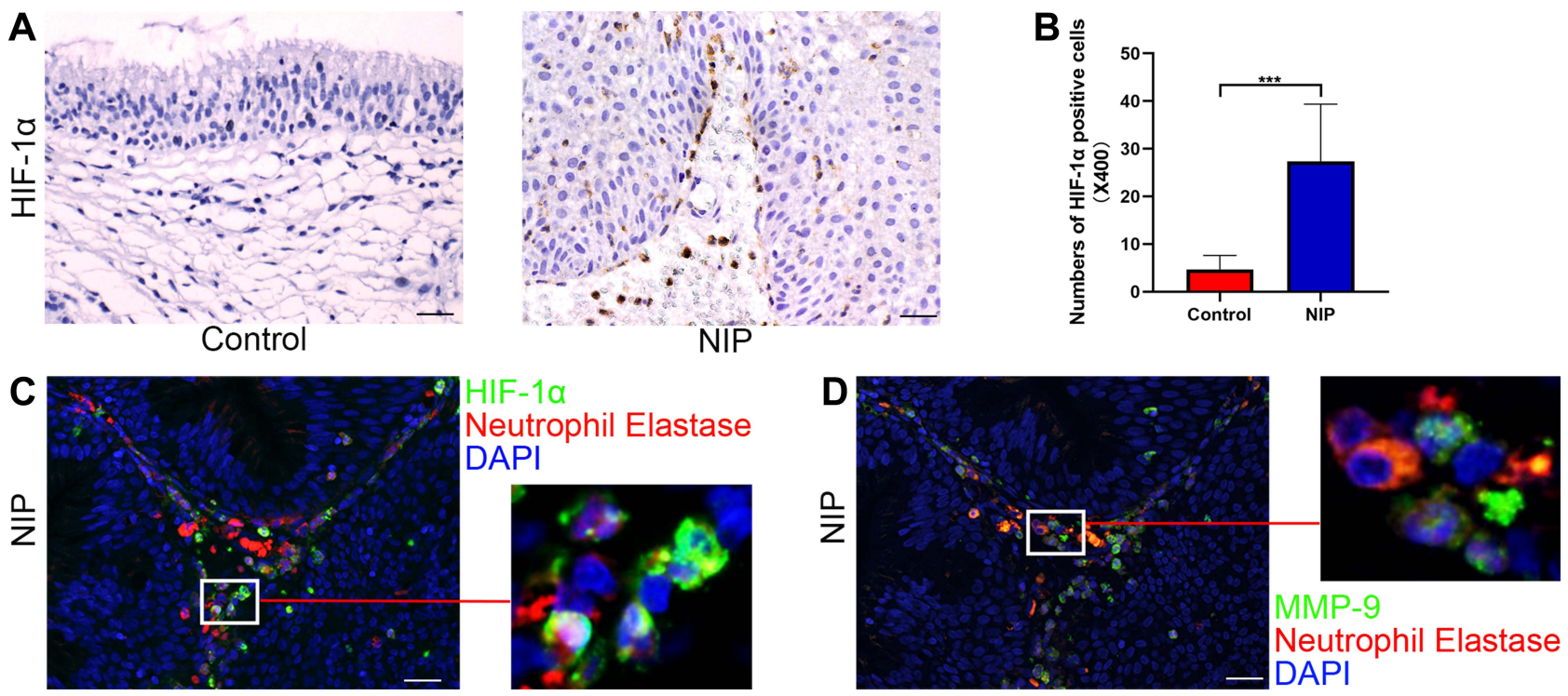

Figure 6 Expression levels of HIF-I $\alpha$ in NIP and its distribution in NIP tissue. $(\mathbf{A})$ immunohistochemical assay of HIF- $\mid \alpha$ in healthy control $(n=I 2)$ and NIP tissue $(n=I 4)$, representative images were shown with the magnification of $\times 400$ (scale bar $=20 \mu \mathrm{m}$ ). (B) HIF-I $\alpha$ positive cell numbers at $\times 400$ magnification from healthy control and NIP tissue. (C and D) co-staining of neutrophil elastase with HIF-I $\alpha /$ MMP-9 in NIP tissue at $\times 400$ magnification (scale bar $=20 \mu \mathrm{m}$ ), higher power field was shown on the right side of each image. Data was shown as mean $\pm \mathrm{SD}$, Statistical significance was calculated using Student's $t$-test, $* * * p<0.00 \mathrm{I}$. 

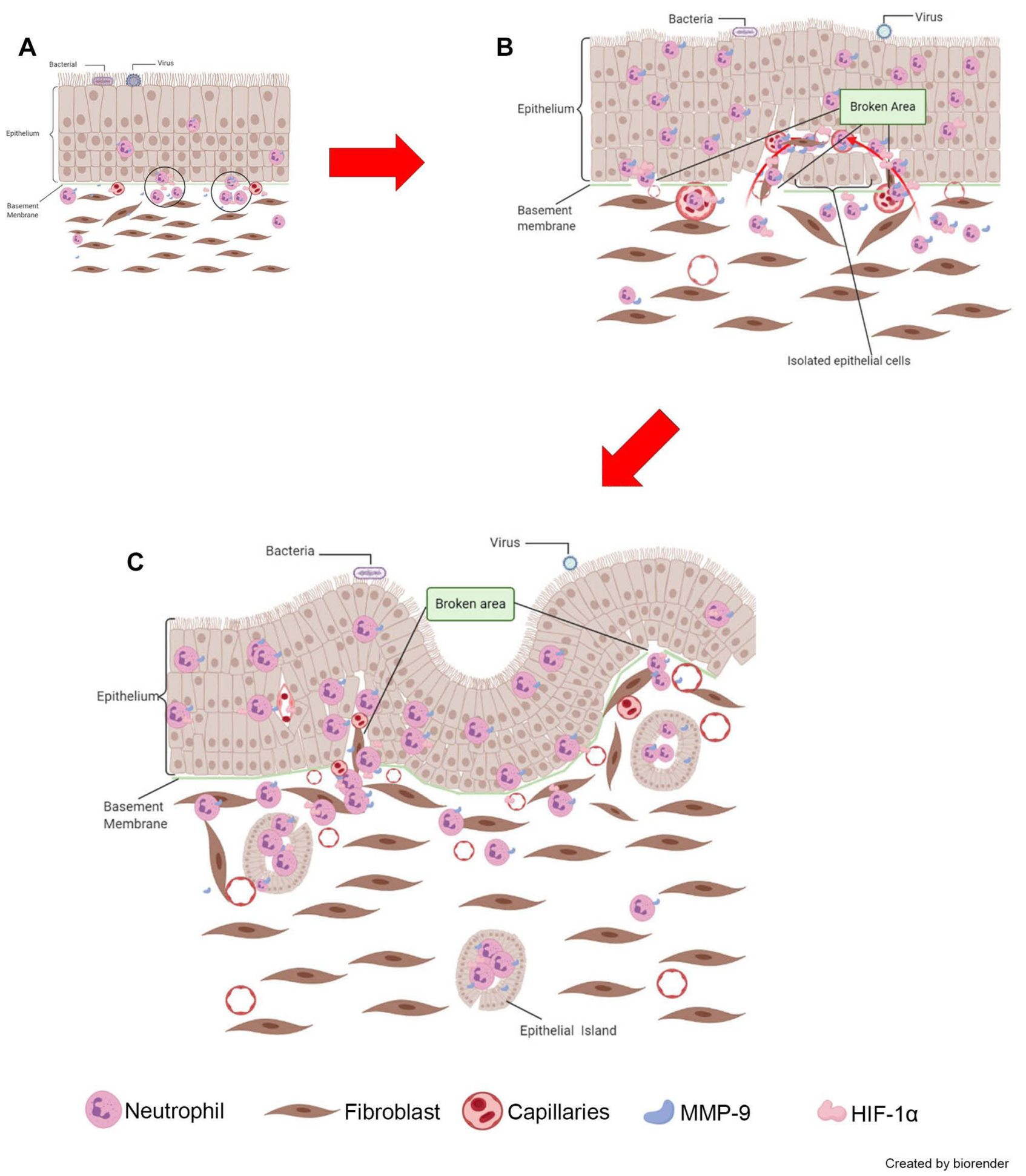

Figure 7 Proposed model of neutrophil and MMP-9's role in the initiation of NIP formation. (A) MMP-9 from infiltrating neutrophils dissolved the basement membrane, leading to inflammatory cells moving into the epithelium. (B) Under the co-effect of HIF-I $\alpha$, the formation of finger-like projections was enhanced. (C) The distortion and formation of NIP and surrounding epithelial islands resulting in the unique histopathological characteristics of NIP. The photos were created by BioRender.com.

process may also allow endothelial cells to move out of the blood vessels to form new capillaries. ${ }^{29}$ Coupled with HIF$1 \alpha$, also a key regulator of angiogenesis, the process hence resulted in enhanced capillary formation that may contribute to the growth of NIP. The newly formed capillaries may also in turn bring more neutrophils to the stroma, resulting in the vicious cycle of NIP pathogenesis and further weakening the basement membrane. The thin and less intact basement 
membrane hence further contributes to the microenvironment of finger-like projections formation and the distortion and disruption of the epithelium.

Besides MMP-9 from neutrophils, we also observed upregulated MMP-1 and MMP-7 in the epithelium of NIP tissue. MMP-7 could regulate cell to cell contacts by loosening adherent junctions' E-cadherin, as well as the migration of cells. ${ }^{30}$ The endothelial adherent junctions can also be cleaved by MMP-7 to increase vascular permeability. ${ }^{31}$ On the other hand, the main substrate of MMP-1 is fibrous collagen, where degradation of collagen fibers and gelatin in the ECM further alters the tumor environment. Hence, up-regulated MMP-1 leads to the degradation of the basement membrane and ECM, which is not conducive to the repair of damaged cells. MMP-1 can also act on the initial stage of tumorigenesis, which contributes to tumor formation. In lung alveolar epithelial cells, MMP-1 can also promote cell proliferation and migration. ${ }^{32}$ Therefore, MMP-1 functions suggest that its upregulation in NIP epithelium may correlate to the hyperplasia of the epithelium. Finally, while only at mRNA levels, MMP-2 reduction in NIP tissue was an interesting finding with its effect unknown and understudied, which warrants future investigation as well.

By combining the results of our study, we speculate that chronic infiltration of neutrophils and impaired clearance by macrophage resulted in prolong neutrophil MMP9 exerting its effect at the stroma, aided by MMP-1 and MMP-7 to compromise the ECM and allow insertion of fibroblast and capillaries into the epithelium (Figure 7B). At the same time, the increased MMP actions were unable to be properly regulated due to the reduction in inhibitor TIMP-1 and TIMP-3 expression. Hence, the cyclical, unique NIP pathogenesis mechanism resulted in fingerlike projection on NIP tissue that becomes a source of epithelial islands constantly existing within the tissue stroma of NIP tissue in the nasal cavity. The formation of these islands is clinically significant as if they are not removed together with the tumor body during surgery, these epithelial islands remaining in the stroma may proliferate again to form new NIPs, which may explain the high recurrence rate of NIP. Based on the mechanisms uncovered from our study, it is proposed that neutrophils or their secreted MMPs may serve as a target in the treatment of NIP formation and recurrence. If we could reduce the neutrophil infiltration, or increase scavenging M2 macrophages, it may lead to the reduction of finger- like projection formation, thus inhibiting, or at least delaying the development and recurrence of NIP.

This study, however, is not without its limitations. Firstly, this is a cross-sectional study that lacks direct comparison between the healthy and NIP region of the same patient. Thus, in the future, sampling of healthy and NIP sites from the same patient would allow for more accurate validation of the factors involved in NIP formation mechanisms identified in this study. In addition, our study only showed observational data and did not provide mechanistic evidence of MMP-9 and HIF-1 $\alpha$ in NIP formation due to the lack of suitable models currently. Future studies utilizing cultures of NIP cells in 3D models with treatment of NIP-associated factors can be explored as a potential model to elucidate these mechanisms.

\section{Conclusion}

In this study, we analyzed the histopathological characteristics of NIP and identified the process that may be crucial in contributing to NIP formation and recurrence. We found that neutrophils congregated in the less intact region of basement membranes where the MMP-9/HIF-1 $\alpha$ released by neutrophils facilitated processes that promote NIP formation. Our study re-affirmed the role of neutrophil infiltration leading to degradation of the basement membrane and initiated the NIP pathogenesis process, resulting in the formation of finger-like projections pathophysiologically unique to NIP. Our study has identified neutrophils and MMPs, particularly MMP-9 as targets in the treatment and recurrence prevention of NIP.

\section{Abbreviations}

NIP, Nasal Inverted Papilloma; MMP, Matrix metalloproteinase; HIF-1 $\alpha$, Hypoxia Induce Factor Alpha; TIMP, Tissue Inhibitor of Metalloproteinase; ECM, Extracellular Matrix; NE, Neutrophils Elastase.

\section{Ethical Statement}

We briefed all participants (NIP patients and healthy controls) in detail on the process of sample collection and all participants provided written informed consent following the declaration of Helsinki. All procedures in this study related to human participants were following the ethical standards of the institutional and/or national research committees' ethical standards. The approval of this study was obtained from the institutional review committee of the participating hospitals (China approval number: KYLL2016 (GJ)P-000). 


\section{Acknowledgment}

This study was supported by the grant National Science Foundation of China (81670909, 81873692), Key R\&D Program of Shandong Province (2018CXGC1214). Dr. Kai Sen Tan is a recipient of fellowship support from the EAACI Research Fellowship 2019.

\section{Disclosure}

The authors declare no conflict of interest.

\section{References}

1. Krouse $\mathrm{JH}$. Endoscopic treatment of inverted papilloma: safety and efficacy. Am J Otolaryngol. 2001;22(2):87-99. doi:10.1053/ ajot.2001.22563

2. Lane AP, Bolger WE. Endoscopic management of inverted papilloma. Curr Opin Otolaryngol Head Neck Surg. 2006;14 (1):14-18. doi:10.1097/01.moo.0000193175.54450.1f

3. Kristensen S, Vorre P, Elbrond O, Sogaard H. Nasal Schneiderian papillomas: a study of 83 cases. Clin Otolaryngol Allied Sci. 1985;10 (3):125-134. doi:10.1111/j.1365-2273.1985.tb01181.x

4. Mirza S, Bradley PJ, Acharya A, Stacey M, Jones NS. Sinonasal inverted papillomas: recurrence, and synchronous and metachronous malignancy. J Laryngol Otol. 2007;121(9):857-864.

5. Zhao L, Li CW, Jin P, et al. Histopathological features of sinonasal inverted papillomas in Chinese patients. Laryngoscope. 2016;126(4): E141-E147. doi:10.1002/lary.25694

6. Reynoso J, Davis RE, Daniels WW, Awad ZT, Gatalica Z, Filipi CJ. Esophageal papillomatosis complicated by squamous cell carcinoma in situ. Dis Esophagus. 2004;17(4):345-347. doi:10.1111/j.14422050.2004.00438.x

7. Zhao Y, Li N, Gong X, Yu L, Jin X. Clinicopathologic features of intraductal papillary neoplasm of breast: analyses of three cases. Int J Clin Exp Pathol. 2017;10(9):9575-9582.

8. Jang HH, Kim HW, Kim SJ, et al. Gastric squamous papilloma in a 52-year-old female patient. Clin Endosc. 2014;47(6):571-574. doi:10.5946/ce.2014.47.6.571

9. Vandenbroucke RE, Libert C. Is there new hope for therapeutic matrix metalloproteinase inhibition? Nat Rev Drug Discov. 2014;13 (12):904-927.

10. Kessenbrock K, Plaks V, Werb Z. Matrix metalloproteinases: regulators of the tumor microenvironment. Cell. 2010;141(1):52-67. doi:10.1016/j.cell.2010.03.015

11. Gross J, Lapiere CM. Collagenolytic activity in amphibian tissues: a tissue culture assay. Proc Natl Acad Sci U S A. 1962;48:1014-1022. doi:10.1073/pnas.48.6.1014

12. Page-McCaw A, Ewald AJ, Werb Z. Matrix metalloproteinases and the regulation of tissue remodelling. Nat Rev Mol Cell Biol. 2007;8 (3):221-233. doi:10.1038/nrm2125

13. Parks WC, Wilson CL, Lopez-Boado YS. Matrix metalloproteinases as modulators of inflammation and innate immunity. Nat Rev Immunol. 2004;4(8):617-629. doi:10.1038/nri1418

14. Deryugina EI, Quigley JP. Matrix metalloproteinases and tumor metastasis. Cancer Metastasis Rev. 2006;25(1):9-34. doi:10.1007/ s10555-006-7886-9

15. Fanjul-Fernandez M, Folgueras AR, Cabrera S, Lopez-Otin C. Matrix metalloproteinases: evolution, gene regulation and functional analysis in mouse models. Biochim Biophys Acta. 2010;1803(1):3-19. doi:10.1016/j.bbamcr.2009.07.004
16. Aimes RT, Quigley JP. Matrix metalloproteinase-2 is an interstitial collagenase. Inhibitor-free enzyme catalyzes the cleavage of collagen fibrils and soluble native type I collagen generating the specific 3/4and 1/4-length fragments. J Biol Chem. 1995;270(11):5872-5876. doi:10.1074/jbc.270.11.5872

17. Mackay AR, Hartzler JL, Pelina MD, Thorgeirsson UP. Studies on the ability of $65-\mathrm{kDa}$ and $92-\mathrm{kDa}$ tumor cell gelatinases to degrade type IV collagen. $J$ Biol Chem. 1990;265(35):21929-21934. doi:10.1016/S0021-9258(18)45827-9

18. Keck T, Balcom JH, Fernandez-del Castillo C, Antoniu BA, Warshaw AL. Matrix metalloproteinase-9 promotes neutrophil migration and alveolar capillary leakage in pancreatitis-associated lung injury in the rat. Gastroenterology. 2002;122(1):188-201. doi:10.1053/gast.2002.30348

19. Chabot V, Reverdiau P, Iochmann S, et al. CCL5-enhanced human immature dendritic cell migration through the basement membrane in vitro depends on matrix metalloproteinase-9. J Leukoc Biol. 2006;79(4):767-778. doi:10.1189/jlb.0804464

20. Okada S, Kita H, George TJ, Gleich GJ, Leiferman KM. Migration of eosinophils through basement membrane components in vitro: role of matrix metalloproteinase-9. Am J Respir Cell Mol Biol. 1997;17 (4):519-528. doi:10.1165/ajrcmb.17.4.2877

21. Papon JF, Lechapt-Zalcman E, Abina M, et al. Matrix metalloproteinase-2 and -9 expression in sinonasal inverted papilloma. Rhinology. 2006;44(3):211-215.

22. Katori H, Nozawa A, Tsukuda M. Increased expression of matrix metalloproteinase-2 and 9 and human papilloma virus infection are associated with malignant transformation of sinonasal inverted papilloma. J Surg Oncol. 2006;93(1):80-85. doi:10.1002/jso.20386

23. Ong CWM, Fox K, Ettorre A, Elkington PT, Friedland JS. Hypoxia increases neutrophil-driven matrix destruction after exposure to Mycobacterium tuberculosis. Sci Rep. 2018;8(1):11475. doi:10.1038/ s41598-018-29659-1

24. Mortaz E, Alipoor SD, Adcock IM, Mumby S, Koenderman L. Update on neutrophil function in severe inflammation. Front Immunol. 2018;9:2171. doi:10.3389/fimmu.2018.02171

25. Chung KF, Adcock IM. Multifaceted mechanisms in COPD: inflammation, immunity, and tissue repair and destruction. Eur Respir J. 2008;31(6):1334-1356. doi:10.1183/09031936.00018908

26. Kettritz R, Xu YX, Kerren T, et al. Extracellular matrix regulates apoptosis in human neutrophils. Kidney Int. 1999;55(2):562-571. doi:10.1046/j.1523-1755.1999.00280.x

27. Kobayashi SD, Voyich JM, Burlak C, DeLeo FR. Neutrophils in the innate immune response. Arch Immunol Ther Exp. 2005;53(6):505-517.

28. Zijlstra A, Seandel M, Kupriyanova TA, et al. Proangiogenic role of neutrophil-like inflammatory heterophils during neovascularization induced by growth factors and human tumor cells. Blood. 2006;107 (1):317-327. doi:10.1182/blood-2005-04-1458

29. Liu Y, Zhang H, Yan L, et al. MMP-2 and MMP-9 contribute to the angiogenic effect produced by hypoxia/15-HETE in pulmonary endothelial cells. J Mol Cell Cardiol. 2018;121:36-50. doi:10.1016/ j.yjmcc.2018.06.006

30. McGuire JK, Li Q, Parks WC. Matrilysin (matrix metalloproteinase-7) mediates E-cadherin ectodomain shedding in injured lung epithelium. Am J Pathol. 2003;162(6):1831-1843. doi:10.1016/S0002-9440(10)64318-0

31. Ichikawa Y, Ishikawa T, Momiyama N, et al. Matrilysin (MMP-7) degrades VE-cadherin and accelerates accumulation of beta-catenin in the nucleus of human umbilical vein endothelial cells. Oncol Rep. 2006;15(2):311-315.

32. Herrera I, Cisneros J, Maldonado M, et al. Matrix metalloproteinase (MMP)-1 induces lung alveolar epithelial cell migration and proliferation, protects from apoptosis, and represses mitochondrial oxygen consumption. J Biol Chem. 2013;288(36):25964-25975. doi:10.1074/ jbc.M113.459784 


\section{Publish your work in this journal}

The Journal of Inflammation Research is an international, peerreviewed open-access journal that welcomes laboratory and clinical findings on the molecular basis, cell biology and pharmacology of inflammation including original research, reviews, symposium reports, hypothesis formation and commentaries on: acute/chronic inflammation; mediators of inflammation; cellular processes; molecular mechanisms; pharmacology and novel anti-inflammatory drugs; clinical conditions involving inflammation. The manuscript management system is completely online and includes a very quick and fair peerreview system. Visit http://www.dovepress.com/testimonials.php to read real quotes from published authors. 